\title{
Is it time to change the goals of lipid management in type 1 diabetes mellitus? Changes in apolipoprotein levels during the first year of type 1 diabetes mellitus. Prospective InLipoDiab1 study
}

Aleksandra Cieluch ${ }^{1}$, Aleksandra Uruska ${ }^{1}$, Marcin Nowicki², Ewa Wysocka ${ }^{3}$, Paweł Niedźwiecki ${ }^{1}$, Agata Grzelka-Woźniak ${ }^{1}$, Justyna Flotyńska ${ }^{1}$, Dorota Zozulińska-Ziółkiewicz ${ }^{1}$

\author{
${ }^{1}$ Department of Internal Medicine and Diabetology, Poznan University of Medical \\ Sciences, Poznan, Poland \\ ${ }^{2}$ Department of Clinical Biochemistry and Laboratory Medicine, Poznan University \\ of Medical Sciences, Poznan, Poland \\ ${ }^{3}$ Department of Laboratory Diagnostics, Poznan University of Medical Sciences, \\ Poznan, Poland
}

Submitted: 18 March 2020; Accepted: 18 July 2020

Online publication: 29 October 2020

Arch Med Sci 2022; 18 (3): 596-603

DOI: https://doi.org/10.5114/aoms.2020.100255

Copyright (c) 2020 Termedia \& Banach

\begin{abstract}
Introduction: Apolipoprotein complement is a critical determinant of lipoprotein function and metabolism. The relation between exogenous insulin and apolipoproteins (apos) in newly diagnosed type 1 diabetes mellitus (T1DM) has not yet been studied extensively. The aim of this study was to prospectively observe the changes in serum apos $\mathrm{Al}$ (apo Al) and All (apo All) in patients with newly diagnosed T1DM and their association with the daily insulin requirement.

Material and methods: Thirty-four participants of the InLipoDiab1 study aged 26 (IQR: 22-32) were enrolled in this analysis. Apolipoprotein Al and All concentrations were assessed at diagnosis and at follow-up after 3 weeks, 6 months, and 1 year of insulin treatment. The daily dose of insulin (DDI) was calculated as the amount of short- and long-acting insulin at discharge from the hospital and at follow-up visits.

Results: The changes in apo Al concentration were observed after 3 weeks of insulin treatment $(p=0.04)$, with the largest increase between 3 weeks and 6 months of observation $(p<0.001)$. Apolipoprotein All level did not change significantly after 3 weeks, while a significant increase was observed between 3 weeks and 6 months of treatment $(p<0.001)$. The correlations between DDI and apo concentration were not statistically significant.

Conclusions: In the first year of T1DM, there is a significant increase in apos concentration. Due to the significant deviation of apos concentration from accepted norms, changes in the recommendations of lipid control criteria in T1DM may be considered.
\end{abstract}

Key words: type 1 diabetes mellitus, apolipoproteins, insulin therapy, HDL metabolism, atherosclerosis.

\section{Introduction}

High-density lipoprotein cholesterol (HDL-C) is the cholesterol fraction responsible for the reverse transport of excess cholesterol from peripheral tissues to the liver, where it is excreted from the body along with

\author{
Corresponding author: \\ Aleksandra Cieluch MD \\ Department of Internal \\ Medicine and Diabetology \\ Poznan University \\ of Medical Sciences \\ Raszeja Hospital \\ 2 Mickiewicza St \\ 60-834 Poznan, Poland \\ Phone: +48 612245270 \\ E-mail: \\ aleksandra.cieluch@o2.pl
}


the bile $[1,2]$. High-density lipoprotein $(\mathrm{HDL})$ is a heterogeneous population of particles differing in shape, size, and composition [3], and is characterized by the presence of group $A$ apolipoproteins (apos) and the absence of apolipoprotein B (apo B). Apolipoprotein complement is a critical determinant of lipoprotein function and metabolism. Apolipoproteins are activators or inhibitors of enzymes and ligands of cellular receptors that play a key role in the conversion of lipoproteins. The protein contained in HDL-C constitutes about $50 \%$ of the composition of the molecule. Apolipoprotein $\mathrm{Al}$ (apo $\mathrm{Al}$ ) is the main protein responsible for the antiatherosclerotic effects of HDL-C [4]. It is the key HDL-C protein, which promotes vasoprotective mechanisms via its ability to promote reverse cholesterol transport from peripheral tissues (i.e., macrophages) to the liver. Next to it, there are apos All (apo All), AIV, C, and E, as well as lipid-transporting enzymes and proteins that play an important role in the metabolism of lipoproteins $[5,6]$. Apolipoprotein All is the second most abundant protein in HDL-C particles. Its role in the reverse transport of cholesterol is controversial. Various observations indicate either the atherosclerotic or anti-atherogenic properties of apo All [7].

People with autoimmune diseases are characterized by the presence of dyslipidemia including reduced HDL-C levels [8-10]. There have been reports on the effect of insulin therapy on HDL-C levels in type 1 diabetes mellitus (T1DM) [11-13], but few studies assessing the effect of insulin on apos. Moreover, there is a lack of data on the relationship between apo abnormalities at the time of T1DM diagnosis and the development of atherosclerotic cardiovascular disease and other complications in the future. Our previous observations showed that the initiation of insulin therapy in patients with newly diagnosed T1DM causes a significant increase in HDL-C level after 3 months of treatment, and this effect is dose-dependent [14]. Conflicting data exist regarding the level of apo Al in patients with T1DM. Researchers have reported decreased, increased, or the same apo Al level compared with healthy controls. Interestingly, the researchers agree that apo All concentration is not significantly different in patients with T1DM compared to the control group [15-18]. An increase in apo Al level was observed along with the duration of the T1DM [19]. Murao et al. showed that insulin stimulates apo Al expression in human HepG2 cells, and this phenomenon is dependent on the dose of insulin. The authors suggested that the ability of insulin to enhance apoA1 gene expression reinforces the recommendation for tight glycemic control to reduce the risk of atherosclerotic disease in T1DM [20]. The data about the relation between insulin therapy and apos in T1DM are limited.
Thus, the study aimed to prospectively observe the changes in serum apos $\mathrm{Al}$ and All in patients with newly diagnosed T1DM and their association with the daily insulin requirement.

\section{Material and methods}

\section{General information}

The Insulin Therapy and Lipoproteins Profile in Type 1 Diabetes Study (InLipoDiab1) is a prospective, observational study. Three hundred patients were recruited for the study so far, but recruitment is still ongoing. Thirty-four patients (22 men and 12 women), aged 26 (IQR: 22-32) with newly diagnosed T1DM were consecutively recruited between June 2015 and October 2017 in the Department of Internal Medicine and Diabetology. All participants of the InLipoDiab1 study are patients with newly diagnosed T1DM who are constantly recruited and regularly participate in visits to the Outpatient Clinic. The current analysis includes people for whom we have collected a set of data at 4 control points, i.e. at the time of diagnosis and after 3 weeks, 6 and 12 months of observation. The inclusion criteria were: new-onset T1DM and treatment with insulin, age between 18 and 35 years old, written consent to participate in the study, no other medical conditions, and no additional drugs apart from insulin. All patients were hospitalized, on average, for 7 days due to newly diagnosed T1DM. The patients were treated with intensive insulin therapy using insulin pens from the moment of diagnosis and underwent the same educational program as we described previously [14]. The daily dose of insulin (DDI) was defined as the requirement for insulin per kilogram of body weight per day. The daily dose of insulin was calculated as the amount of short and long-acting insulin (basal + bolus) at discharge from the hospital and at follow-up visits in the outpatient clinic from the doses of insulin noted by the patients in their self-monitoring of blood glucose logs 1 day before the visit. The autoimmune etiology was confirmed in all patients by the positive specific autoantibodies: islet cell antibodies (a-ICA), glutamic acid decarboxylase antibodies (a-GAD), and tyrosine phosphatase antibodies (a-IA2). The study is registered at ClinicalTrials.gov (NCT02306005). The study followed the tenets of the Declaration of Helsinki and was approved by the institutional ethics committee, with written informed consent from each participant. All data were obtained from a face-to-face interview, physical examination, and biochemical measurement. Blood pressure was measured with an arm type manual sphygmomanometer one 3 times after resting for more than $10 \mathrm{~min}$, and then the average was taken. Characteristics of the study group at diagnosis are presented in Table I. 
Table I. Characteristics of the study group at diagnosis. Results are presented as median values and interquartile range $(I Q R)$ or number and percentage

\begin{tabular}{|c|c|c|c|}
\hline Variable & $\begin{array}{l}\text { All patients } \\
\qquad N=34\end{array}$ & $\begin{array}{l}\text { Women } \\
n=12\end{array}$ & $\begin{array}{c}\text { Men } \\
n=22\end{array}$ \\
\hline Age at diagnosis [years] & $26(22-32)$ & $24(22-31)$ & $31(28-35)$ \\
\hline $\begin{array}{l}\text { DDI at discharge from hospital at } \\
\text { diagnosis [units } / \mathrm{kg} / \mathrm{day} \text { ] }\end{array}$ & $0.12(0.09-0.19)$ & $0.13(0.11-0.26)$ & $0.10(0.08-0.17)$ \\
\hline $\begin{array}{l}\text { Body mass before the diagnosis } \\
\text { of diabetes }[\mathrm{kg}]^{\star}\end{array}$ & $77.30(61.60-87.50)$ & $58.75(55.60-66.90)$ & $84.4(76.3-88.3)$ \\
\hline Body mass at diagnosis [kg] & $69.15(56.60-82.30)$ & $53.05(49.60-57.00)$ & $78.2(70.00-85.00)$ \\
\hline $\mathrm{BMI}\left[\mathrm{kg} / \mathrm{m}^{2}\right]$ & $22.29(19.56-23.63)$ & $19.40(18.66-21.49)$ & $23.21(21.89-26.55)$ \\
\hline Waist circumference $[\mathrm{cm}]$ & $80(70-86)$ & $66.50(64-73)$ & $83(80-93)$ \\
\hline WHR & $0.80(0.75-0.87)$ & $0.74(0.71-0.77)$ & $0.85(0.80-0.91)$ \\
\hline $\mathrm{SBP}[\mathrm{mm} \mathrm{Hg}]$ & $120(110-127)$ & $118(110-121)$ & $121(115-132)$ \\
\hline $\mathrm{DBP}[\mathrm{mm} \mathrm{Hg}]$ & $80(70-87)$ & $80(70-86.5)$ & $80(70-87)$ \\
\hline Fat tissue content (\%) & $19.35(13.55-22.30)$ & $20.6(17.6-26.1)$ & $18.0(10.4-21.3)$ \\
\hline MFG $[\mathrm{mmol} / \mathrm{l}]$ & $7.44(6.57-7.86)$ & $7.4(6.6-7.6)$ & $7.45(6.57-8.06)$ \\
\hline MPG [mmol/l] & $9.33(8.41-10.08)$ & $8.98(8.29-9.77)$ & $9.36(8.69-10.11)$ \\
\hline Creatinine $[\mu \mathrm{mol} / \mathrm{l}]$ & $72.49(64.53-83.10)$ & $64.53(56.58-66.30)$ & $82.21(70.72-90.17)$ \\
\hline AIAT [U/I] & $23.00(16.00-31.00)$ & $17.00(10.00-30.50)$ & $24.00(17.00-31.00)$ \\
\hline $\mathrm{HbA}_{1 \mathrm{c}}(\%)$ & $11.15(9.50-12.30)$ & $11.7(9.45-13.00)$ & $10.8(9.5-11.9)$ \\
\hline Total cholesterol [mmol/l] & $4.51(3.80-5.04)$ & $4.22(3.75-4.65)$ & $4.58(3.85-5.74)$ \\
\hline HDL cholesterol [mmol/l] & $1.22(1.01-1.40)$ & $1.23(1.18-1.69)$ & $1.19(0.96-1.34)$ \\
\hline LDL cholesterol [mmol/l] & $2.58(2.00-3.55)$ & $2.31(1.85-2.76)$ & $2.97(2.27-4.00)$ \\
\hline Triglycerides [mmol/l] & $1.21(0.94-1.60)$ & $1.11(0.80-1.43)$ & $1.21(0.99-2.31)$ \\
\hline $\mathrm{TSH}[\mu \mathrm{IU} / \mathrm{ml}]$ & $1.86(1.26-2.92)$ & $2.25(1.12-3.04)$ & $1.81(1.35-2.62)$ \\
\hline
\end{tabular}

${ }^{\star}$ Data from medical history. DDI - daily dose of insulin, BMI - body mass index, WHR - waist-hip ratio, SBP - systolic blood pressure, $D B P$ - diastolic blood pressure, MFG - mean fasting glucose, MPG - mean postprandial glucose, AlAT - alanine transaminase, $H b A_{1 c}$ - glycated hemoglobin, HDL - high-density lipoprotein, LDL - low-density lipoprotein, TSH - thyroid stimulating hormone.

\section{Anthropometric data}

Physical examination included the measurements of body weight, height, fat tissue content, waist, and hip circumference. Body weight and fat tissue content were measured by Body Composition Analyzer Tanita. Height, waist, and hip circumference were measured by trained investigators using standardized meter measures. The body mass index (BMI) and waist-to-hip ratio (WHR) were calculated from the following formulas: $\mathrm{BMI}=$ weight $(\mathrm{kg}) /$ squared height $\left(\mathrm{m}^{2}\right)$ and WHR = waist circumference $(\mathrm{cm}) /$ hip circumference $(\mathrm{cm})$, respectively.

\section{Diabetes mellitus metabolic control}

The measurement of glycated hemoglobin $\left(\mathrm{HbA}_{1 \mathrm{c}}\right)$ concentration was made in whole blood and assessed by a turbidimetric inhibition immunoassay (Cobas 6000, Roche Diagnostics). Mean fasting glucose (MFG) and mean postprandial glucose (MPG) were measured using capillary plasma with a glucometer. Mean postprandial glucose was measured 3 times, $2 \mathrm{~h}$ after major meals throughout the day. Mean fasting glucose and MPG were expressed as the mean of patients' measurements from all days of hospital stay.

\section{Assessment of lipid profile and apolipoproteins}

The measurement of apo Al and apo All concentration was conducted at 4 time points: at the time of diagnosis (before administration of insulin) and at follow-up after 3 weeks, 6 months and 1 year of insulin treatment. The concentration of apo $\mathrm{Al}$ and apo All was determined with AssayPro company tests using the immunoenzymatic method ELISA (enzyme-linked immunosorbent assay) using a TECAN-SUNRISE reader 
with Magellan software. Apolipoprotein Al/apo All ratio was calculated by dividing apo Al by apo All. The evaluation of the lipid profile was conducted at the time of diagnosis (before administration of insulin). High-density lipoprotein cholesterol, total cholesterol (TC), and triglyceride (TG) levels were measured with the Cobas 6000 biochemistry analyzer (Roche Diagnostics) using enzymatic colorimetric methods. All of the above measurements were performed in serum. Low-density lipoprotein cholesterol level was calculated by the Friedewald formula. NonHDL-C was calculated as TC minus HDL-C. Triglyceride/HDL-C ratio was calculated by dividing TG by HDL-C (in mg/dl units). To properly assess the effect of insulin therapy on apos, the inclusion criterion was to obtain baseline blood samples of the patients immediately after the diagnosis of diabetes and before administration of the first insulin injection.

\section{Statistical analysis}

Statistical analysis of results was performed using Statistica v. 13.0 software (StatSoft, Tulsa, CA, USA). The Kolmogorov-Smirnov test with Lilliefors correction was used to analyze the normal distribution of data. Non-parametric tests were used for further analyses because analyzed parameters did not have a normal distribution. Results were presented as numbers and percentages, as well as medians and interquartile ranges (IQR). ANOVA Friedman test was used to analyze differences between subgroups for numerical variables. A comparison of selected parameters at 2 different points of observation was tested by the Wilcoxon test. Spearman correlation was used to evaluate the association between the DDI and Apo Al and Apo All concentration. $P<0.05$ was considered statistically significant.

\section{Results}

\section{Apolipoproteins}

Significant increases in apo $\mathrm{Al}$ and apo All concentrations were revealed during the first year of insulin therapy. The changes in apo Al concentration were observed after the first 3 weeks of intensive insulin therapy, with the largest increase between 3 weeks and 6 months of observation. The same trend was found in the apo Al/apo All ratio. In contrast, apo All levels did not change significantly after 3 weeks of treatment, while a significant increase was observed between 3 weeks and 6 months of treatment. Between 6 and 12 months of the study, we observed a further increase in the level of apo Al and a decrease in

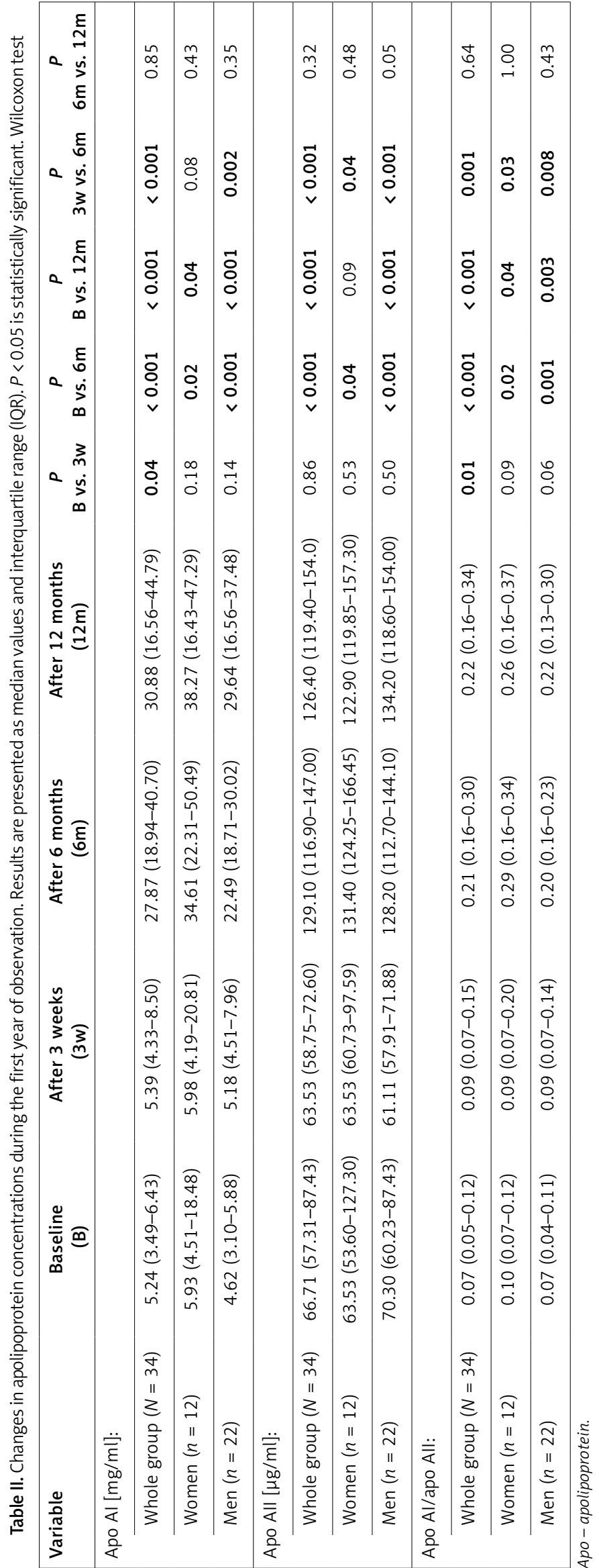


A

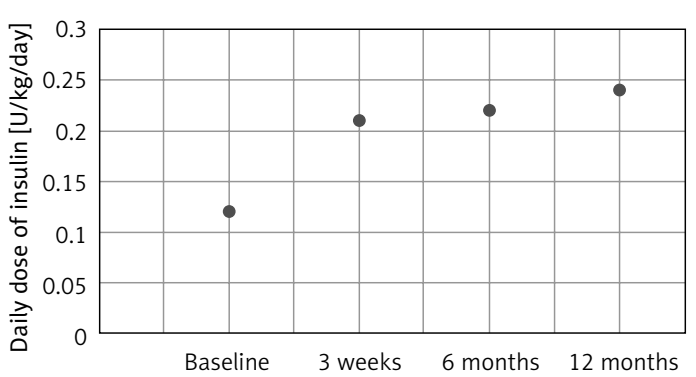

B

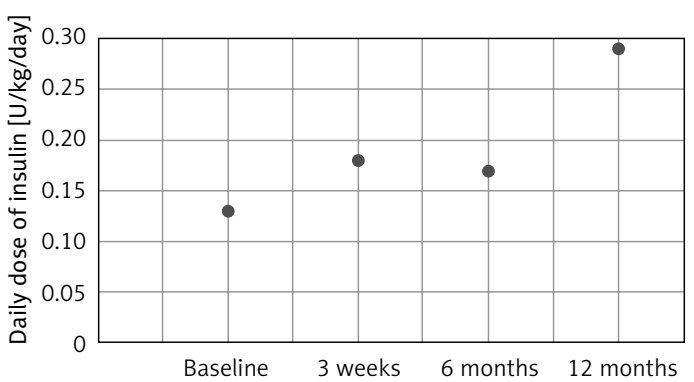

C

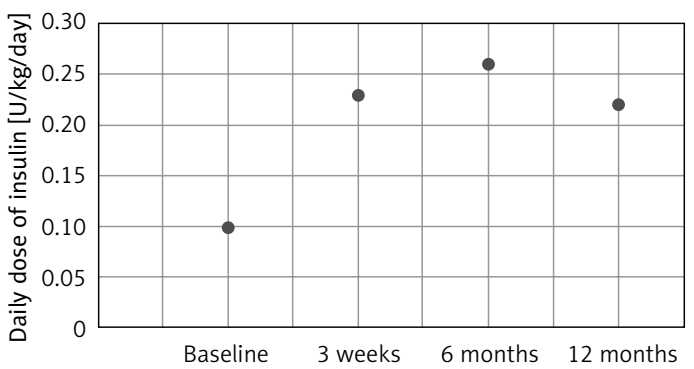

Figure 1. Daily dose of insulin during 12 months of follow-up. A - All group, $p=0.03$, B - women, $p=0.36, \mathbf{C}-$ men, $p=0.008$ (ANOVA Friedman test). Results are presented as median values

the level of apo All, but these changes were not statistically significant. The correlations between DDI and apo concentration also were not statistically significant. Changes in apolipoprotein concentration for the whole group and according to sex are shown in Table II, while changes in DDI are shown in Figure 1.

\section{Discussion}

The obtained results provide first-line evidence showing dynamic changes in the concentration of apos Al and All, during the first year of intensive insulin therapy in patients with newly diagnosed T1DM. The results indicate a significant increase in anti-atherogenic apo Al and probably proatherogenic apo All in the first year of disease duration. However, we have not proven the direct effect of a DDI on the increase in apos con- centration. Surprisingly, our study demonstrated that apo Al level significantly exceeds accepted norms, both at the time of diagnosis and after 12 months of the study. On the other hand, apo All level (despite the significant increase in concentration) is significantly below accepted norms at the same time points [21, 22]. The above results suggest a protective mechanism against metabolic disturbance which occurs at the onset of T1DM. This shows how important it is to achieve good management of the disease from the beginning in order to improve the outcome and prevent the development of complications in the future.

Lipid disorders have a proven impact on the development of chronic complications, both in T1DM and type 2 diabetes mellitus (T2DM). Patients with T1DM are characterized by higher HDL-C levels, but their life expectancy is shorter than their peers [23]. We assume that despite high HDL-C levels, its qualitative composition may show abnormalities and function may be impaired, which predicts development of diabetic complications [24]. During our observation, we revealed an increase in the concentration of anti-atherogenic apo Al and apo All, whose role in atherogenesis is unknown. Studies on animals showed that apo All promotes insulin resistance and has diverse effects on fat homeostasis. Apolipoprotein All transgenic mice had increased adipose mass, and higher plasma leptin and insulin levels than control mice. Moreover, these mice exhibited a delay in plasma clearance of a glucose bolus. Adipose tissue from fasted apo All transgenic mice was characterized by a $50 \%$ decrease in triglyceride hydrolysis compared with control mice. Skeletal muscle from fasted apo All transgenic mice exhibited reduced uptake of 2-deoxyglucose compared with control mice [25]. It might be possible that the development of complications and premature death is associated with adverse changes in apo All already during the first year of T1DM. This may indicate that not only the concentration but also the composition of HDL may be pivotal in T1DM, probably by changing its function.

Our observations regarding the increase in the concentration of apo Al are consistent with the Wilson et al. study. In a relatively small group of young patients with T1DM, they revealed that insulin therapy intensification results in increased apo Al concentration after 3 and 6 months of treatment [11]. Intensification of treatment consisted of transition from the model of twice-daily insulin doses consisting of NPH insulin and regular insulin to the method of multiple injections consisting of regular insulin given before meals (doses were given as units per calorie of ingested food) and a subcutaneous small dose of NPH insulin given at bedtime according to individual requirement, as estimated by the fasting blood glucose level. Our pa- 
tients were treated with intensive insulin therapy from the beginning, and the insulin dose increased as the study progressed, but we did not observe the direct effect of the insulin dose on apos levels.

Other prospective studies assessing apos in T1DM are not consistent with our observations. Kobbah et al. [26] similarly to us assessed lipid parameters prospectively, but the observation time was longer (2 years) and the population consisted of children with newly diagnosed T1DM treated with intravenous insulin infusion for the first 48-72 $\mathrm{h}$ and then with 2 doses of combinations of regular and intermediate insulins. Concentrations of apo $\mathrm{Al}$ and apo All (more pronounced in apo $\mathrm{Al}$ ), and apo $\mathrm{Al} /$ apo All ratio were increased on admission to the hospital in comparison with healthy controls but were restored to normal during 2-year insulin treatment. The results obtained in children are puzzling because, in our study, in the adult population, we observed quite the opposite phenomenon.

Fukui et al., in their non-prospective study, showed that patients with T2DM treated with diet and exercise therapy, either alone or in combination with antihyperglycemic agents, except insulin, had significantly lower apo Al levels in comparison with patients with T1DM and T2DM treated with multiple injections of insulin in a basal-bolus manner. In the same study there were no differences in apo All level. The authors pointed to the effect of insulin on apos. However, there was no significant difference in apo Al level between T1DM and T2DM with insulin, while the total dose of injected insulin was higher in T1DM than in T2DM [27].

Our observations concern changes in apos $\mathrm{Al}$ and All at several points during the first year of the disease. A small study on patients with newly diagnosed T1DM with severe insulinopenia revealed no significant changes in apo $\mathrm{Al}$ concentrations during the first 5 days of insulin therapy, but significant normalization in the lipoprotein profile at 2 days after initiation of insulin therapy. Thus, the results of this study showed that insulin caused changes in lipoprotein levels earlier than in the apos levels [28]. Our previous observations have shown that dynamic changes in HDL-C levels occur in the first year of T1DM and insulin therapy. A significant increase appeared after 3 months of insulin therapy and progressed over time, which we also observed between 3 and 12 months of treatment. This is in contrast to changes in apo Al and apo All, in which we did not record significant changes between 6 and 12 months of T1DM.

Apolipoproteins Al and All have been associated with chronic complications of T1DM. Apolipoprotein All level is positively associated with common and/or internal carotid intima-media thickness (IMT) in men, while apo Al level is inversely associated with internal carotid IMT in women [29]. Concerning diabetic nephropathy, the data are ambiguous. Both elevated levels of apo Al and apo All, as well as reduced levels of these apos, are associated with the development of chronic renal complications [30-32]. In contrast, another study did not reveal differences in apo $\mathrm{Al}$ and apo All in patients with normo-, micro-, and macroalbuminuria [33]. In the case of diabetic retinopathy, an increased, decreased, or unchanged level of apo Al was observed in diabetics with retinopathy compared to patients without the complication or healthy subjects [34-36]. Thus, there are conflicting data on the relationship between apos level and complications in T1DM, perhaps due to different stages of the disease, duration of diabetes or DDI.

The available results of research on apos level do not provide knowledge about their dynamic changes resulting from the initiation of insulin therapy in the first year of diabetes, and there is a lack of new studies on this phenomenon. Our findings may elucidate the role of exogenous insulin in lipid metabolism and prospectively support previous evidence that its disturbances influence diabetic complications.

However, our study has some limitations. We conducted a study on a relatively small group with a short time of observation. Furthermore, no electrophoretic separation of lipoproteins was performed. However, further analyses in this group are planned.

In conclusion, serum apo Al and apo All concentration increased significantly in patients with newly diagnosed T1DM during the first year of diagnosis and treatment. A clear increase in apos concentration appears 6 months after diagnosis and remains stable after 12 months of treatment. The obtained results can become a strong background for further analysis, because we suggest that the profile of serum apos beside traditional lipid level may be a prognostic marker of macro- and microvascular dysfunction and may play a role in the development of chronic complications in T1DM. Due to the significant deviation of apos concentration from accepted norms, changes in the recommendations of lipid management criteria in T1DM may be considered.

\section{Acknowledgments}

The study was funded by the Poznan University of Medical Sciences, Faculty of Medicine II (Grant for Young Scientists number 502-14-0223 4382-99658).

\section{Conflict of interest}

The authors declare no conflict of interest. 


\section{References}

1. Lund-Katz S, Phillips MC. High density lipoprotein structure-function and role in reverse cholesterol transport. Subcell Biochem 2010; 51: 183-227.

2. Gillard BK, Rosales C, Xu B, Gotto AM Jr, Pownall HJ. Rethinking reverse cholesterol transport and dysfunctional high-density lipoproteins. J Clin Lipidol 2018; 12: 849-56.

3. Martin SS, Jones SR, Toth PP. High-density lipoprotein subfractions: current views and clinical practice applications. Trends Endocrinol Metab 2014; 25: 329-36.

4. Mangaraj M, Nanda R, Panda S. Apolipoprotein A-I: a molecule of diverse function. Indian J Clin Biochem 2016; 31: 253-9.

5. Dominiczak MH, Caslake MJ. Apolipoproteins: metabolic role and clinical biochemistry applications. Ann Clin Biochem 2011; 48: 498-515.

6. Zhang T, Tang X, Mao L, et al. HDL-associated apoCIII plays an independent role in predicting postprandial hypertriglyceridemia. Clin Biochem 2020; S0009-9120: 31197-X.

7. Tailleux A, Duriez P, Fruchart JC, Clavey V. Apolipoprotein A-II, HDL metabolism and atherosclerosis. Atherosclerosis 2002; 164: 1-13.

8. Pietrzak A, Chabros P, Grywalska E, et al. Serum lipid metabolism in psoriasis and psoriatic arthritis - an update. Arch Med Sci 2019; 15: 369-75.

9. Toms TE, Panoulas VF, Kitas GD. Dyslipidaemia in rheumatological autoimmune diseases. Open Cardiovasc Med J 2011; 5: 64-75.

10. Yücel Ç, Omma A, Sertoğlu E, Sezer S, Turhan T, Özgürtaş T. Evaluation of atherogenic laboratory markers in Behçet's disease patients with vascular involvement. Arch Med Sci 2019; 16: 531-7.

11. Wilson DP, Fesmire JD, Endres RK, Blackett PR. Increased levels of HDL-cholesterol and apolipoprotein A-I after intensified insulin therapy for diabetes. South Med J 1985; 78: 636-8

12. Guy J, Ogden L, Wadwa RP, et al. Lipid and lipoprotein profiles in youth with and without type 1 diabetes: the SEARCH for Diabetes in Youth case-control study. Diabetes Care 2009; 32: 416-20.

13. Sinha A, Formica C, Tsalamandris C, et al. Effects of insulin on body composition in patients with insulindependent and non-insulin-dependent diabetes. Diabet Med 1996; 13: 40-6.

14. Cieluch A, Uruska A, Grzelka A, Zozulińska-Ziółkiewicz D. An increase in high-density lipoprotein cholesterol concentration after initiation of insulin treatment is dose-dependent in newly diagnosed type 1 diabetes. The results of the InLipoDiab1 study. Pol Arch Intern Med 2018; 128: 960-2.

15. al-Muhtaseb N, al-Yusef AR, Bajaj JS. Lipoprotein lipids and apolipoproteins (AI, All, B, CII, CIII) in type 1 and type 2 diabetes mellitus in young Kuwaiti women. Diabet Med 1991; 8: 732-7.

16. Colhoun HM, Taskinen MR, Otvos JD, Van Den Berg P, O'Connor J, Van Tol A. Relationship of phospholipid transfer protein activity to $\mathrm{HDL}$ and apolipoprotein B-containing lipoproteins in subjects with and without type 1 diabetes. Diabetes 2002; 51: 3300-5.

17. Briones ER, Mao SJ, Palumbo PJ, O’Fallon WM, Chenoweth W, Kottke BA. Analysis of plasma lipids and apolipoproteins in insulin-dependent and noninsulin-dependent diabetics. Metabolism 1984; 33 42-49.

18. Taskinen MR, Kahri J, Koivisto V, Shepherd J, Packard CJ. Metabolism of HDL apolipoprotein A-I and A-II in type 1 (insulin-dependent) diabetes mellitus. Diabetologia 1992 35: 347-56.

19. Krawczuk-Rybak M, Wójtowicz J, Urban M. Lipid metabolism in patients with insulin dependent diabetes. IV. Effect of sex, age, excess body weight, duration of diabetes, insulin dosage and family history on lipid metabolism in patients with insulin dependent diabetes. Endokrynol Pol 1992; 43: 125-35.

20. Murao K, Wada Y, Nakamura T, Taylor AH, Mooradian AD, Wong NC. Effects of glucose and insulin on rat apolipoprotein A-I gene expression. J Biol Chem 1998; 273 : 18959-65.

21. Contois J, McNamara JR, Lammi-Keefe C, Wilson PW, Massov T, Schaefer EJ. Reference intervals for plasma apolipoprotein A-1 determined with a standardized commercial immunoturbidimetric assay: results from the Framingham Offspring Study. Clin Chem 1996; 42: 507-14.

22. Blanco-Vaca F, Escolà-Gil JC, Martín-Campos JM, Julve J. Role of apoA-II in lipid metabolism and atherosclerosis: advances in the study of an enigmatic protein. J Lipid Res 2001; 42: 1727-39.

23. Mäkinen VP, Forsblom C, Thorn LM, et al. Metabolic phenotypes, vascular complications, and premature deaths in a population of 4,197 patients with type 1 diabetes. Diabetes 2008; 57: 2480-7.

24. Costacou T, Evans RW, Orchard TJ. High density lipoprotein cholesterol in diabetes: is higher always better? J Clin Lipidol 2011; 5: 387-94.

25. Castellani LW, Goto AM, Lusis AJ. Studies with apolipoprotein A-II transgenic mice indicate a role for HDLs in adiposity and insulin resistance. Diabetes 2001; 50: 643-51.

26. Kobbah M, Vessby B, Tuvemo T. Serum lipids and apolipoproteins in children with type 1 (insulin-dependent) diabetes during the first two years of the disease. Diabetologia 1988; 31: 195-200.

27. Fukui T, Hirano T. High-density lipoprotein subspecies between patients with type 1 diabetes and type 2 diabetes without/with intensive insulin therapy. Endocr J 2012; 59: 561-9.

28. Reverter JL, Sentí M, Rubiés-Prat J, et al. Lipoprotein composition in the insulin-deficient non-acidotic phase of type I diabetic patients and early evolution after the start of insulin therapy. Clin Chim Acta 1993; 223: 113-20.

29. Basu A, Jenkins AJ, Stoner JA, et al. Apolipoproteindefined lipoprotein subclasses, serum apolipoproteins, and carotid intima-media thickness in T1D. J Lipid Res 2018; 59: 872-83.

30. Jenkins AJ, Yu J, Alaupovic P, et al. Apolipoprotein-defined lipoproteins and apolipoproteins: associations with abnormal albuminuria in type 1 diabetes in the diabetes control and complications trial/epidemiology of diabetes interventions and complications cohort. J Diabetes Complications 2013; 27: 447-53.

31. Tolonen N, Forsblom C, Thorn L, et al. Lipid abnormalities predict progression of renal disease in patients with type 1 diabetes. Diabetologia 2009; 52: 2522-30.

32. Soedamah-Muthu SS, Colhoun HM, Taskinen MR, IdziorWalus B, Fuller JH, Group EICS. Differences in HDLcholesterol: apoA-I + apoA-II ratio and apoE phenotype with albuminuric status in type I diabetic patients. Diabetologia 2000; 43: 1353-9.

33. Kahri J, Groop PH, Elliott T, Viberti G, Taskinen MR. Plasma cholesteryl ester transfer protein and its relationship to plasma lipoproteins and apolipoprotein A-I-containing lipoproteins in IDDM patients with 
microalbuminuria and clinical nephropathy. Diabetes Care 1994; 17: 412-9.

34. Maioli M, Tonolo G, Pacifico A, et al. Raised serum apolipoprotein (a) in active diabetic retinopathy. Diabetologia 1993; 36: 88-90.

35. Simó R, Higuera M, García-Ramírez M, Canals F, GarcíaArumí J, Hernández C. Elevation of apolipoprotein A-I and apolipoprotein $\mathrm{H}$ levels in the vitreous fluid and overexpression in the retina of diabetic patients. Arch Ophthalmol 2008; 126: 1076-81.

36. Sasongko MB, Wong TY, Nguyen TT, et al. Serum apolipoproteins are associated with systemic and retinal microvascular function in people with diabetes. Diabetes 2012; 61: 1785-92. 